\title{
Observations sur les dépôts de boue dans la galerie d'amenée de la centrale de Bévercé
}

\section{Observations on deposits of mud in the head-race channel at Beverce power station}

\author{
PAI (․ THIBESSARD

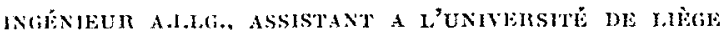

\begin{abstract}
Lobservation des photographies et des monlages et lanalyse des échantillons pris à l'intérient de cette galerie, it troneons métalliques, bètonnés ou rocheux, après plusieurs années de service, montrent un dépót a stratification quasi annuelle, da prédominance manganeuse, de conlenr brune, d'épaisseur moyenne relativement ronstante, formant des argues perpendiculaires a l'écoulement et dont la Iongueur d'onde parie anec l'épaisseur. Traces de charriage et aspect plus lisse à lintérieur des coudes ét dans les divergents. Analogie avec les rides des plages de sable et les dunes du désert. Relation linéaire possible entre l'épaisseur du dépót et la hanteur de la vague, de la créte au creux; infuence éventuelle de la vitesse d'écoulement sur la longuetur d'onile.
\end{abstract}

\begin{abstract}
Examination of pholographs and of castings and the analysis of samples, taken inside this tunnel, of metal, concrete, or rock sections rifer several years scrvice, show a deposit made up of layers formed almost yearly; the substance is brown and is mainly of munganous malerial; the average thickness is comparatively constant and it forms waves, perpendicular to the flow whose length varies with the thickness. Traces of drifting and a smoother appearance on the inside of the bends and in the branches. Analogy with ridges on sandy beaches and with sand dunes in a desert. Possible linear relationship between thickness of the deposit and height of the wave from the crest to the trough. Possible influence of the speed of flow on the length of the ware.
\end{abstract}

\section{INTRODUCTION}

La galerie d'adduction de la centrale de Bévercé a déjà fait l'objet d'une communication de MM. Schlik et Simoss à la tribune de la S.H.F. (1) (*). Celte communication avait Irait aux rariations de la perte de charge de la galerie en fonction du temps. Ces variations, attribuées au dépôt qui se forme dans la conduite, étaient cependant beaucoup trop importantes pour etre dues à la seule diminution de section utile. De 0,0202 en 1932 , la valeur de $\lambda$ avait atteint 0,0263 en 1949 pour retomber à 0,0154 immédiatement après le nettoyage de 1949. D'après les souvenirs du personnel, le dépôt boueux présentait des ondulations en forme de ragues. Ces ondulations

(*) Les chifres entre parentheses renvoient a la notice bibliographique. avaient été rapprochées de celles étudiées par Schlichting (2) et il semblait que la correspondance pouvait être considérée comme satisfaisante.

Des observations analogues avaient également été faites en Allemagne, sur la conduite de l'Ecker (3).

Désireux de se former une idée exacte de ce qu'était réellement le dépôt boueux, MM. Schlati et Simons avaient demandé à visiter la conduite lorsqu'elle serait à nouveau mise à sec.

L'entretien normal des troncons mélalliques de la galerie d'amenée ayant nécessité la vidange de la conduite à la fin de l'été, la société exploilante a très aimablement invité MM. ScHLAr et Simons, peu de temps aprés la mise à sec.

Nous avons ainsi eu l'occasion de visiter les 
conduites à une période tris intéressante; les tronçons mélalliques n'avaient plus ité touches depuis 1934-1937, les troncons cimentés ou rocheux avaient par contre élé nelloyés en 1949 , ansi que certaines parties métalliques. C'est à l'occasion de cette visite que $M$. Schlar nous a demandé de réunir le plus d'observations possibles.

Disons tout de suite que ces observalions se rapportent uniquement a l'aspect de la galerie ef qu'aucune mesure de perte de charge ou de débit n'a pu être faitc.

\section{Les visites}

Le 10 septembre 1953, nous avons visite les tronçons métalliques qui n’avaient plus été touchés depuis 1934-1936, ainsi que la galerie en ciment qui avait été nettoyée en 1949. De cette visite nous avons rapporté trois photos. Nous avons également prélevé des achantillons de dépôt en différents endroils des galeries visitíes, aux fins d'analyse chimique el biologique.

Afin de conserver un témoin matériel du relief du dépòt, nous avons pensé à en effectuer le moulage. Munis du matériel nécessaire, nous nous sommes rendus à Bévercé le 17 septembre 1953. Nous arons procédé à deux moulages en place, l'un dans la galerie métallique, fautre dans la galerie en ciment. Avant de procéder au moulage, nous avons photographié le dépôt. Le même jour, nous avons parcouru des tronçons rocheux de la galerie. Une photo prise dans cette section n'a malheureusement pas réussi, le photo-flash dont nous disposions n'élant pas assez puissant. De cette visite, nous avons encore rapporté aux fins d'analyse des échantillons de dépôt, lant de la partie rocheuse que de la partie métallique el de la partie cimentéc.

Nous nous sommes rendus sur place une derniere fois le 2 octobre 1953. Nous avons visité un tronçon métallique qui n'avait plus été touché depuis 1935-1937. Nous y avons pris trois moulages dont nous avons préalablement photosraphié l'emplacement.

La position des photos, prélèvements el moulages a été repérée sur le schéma de la jigure 1.

\section{Les observations visuelles}

Pour alléger nolre expose, nous utiliserons les notations suivantes (fig. 2):

I. longueur d'onde: distance moyenne sciparanl deux crêtes successives;

$h$, hauteur des vagues ou des ondulations : distance moyenne entre les crenx et les crôles; r, épaisseur du dépòt : distance moyenne entre la paroi el le sommel des cretes, diminué de $h / 2$.

Le dépôt a une couleur brune lrès foncé, presque noire. Il est tres glissant el luisant en surfice quand il est humide; sec, il se résoul en une poussière tris fine. Il n'adhere pas a la paroi et ne se pétrifie pas. Son épaisseur est relativement constante, elle diminue cependant à proximité du sommet de la galerie. Nous n'avons pas pu savoir si elle augmentait dans le font, le joassage des ouviers occupés au curelage ayant

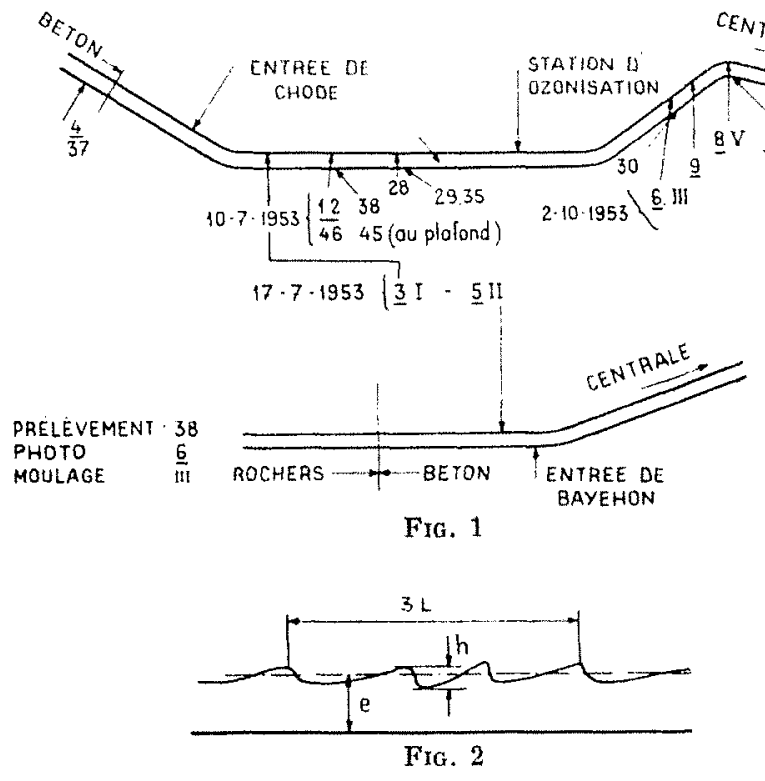

complètement bouleversti le dépòt. Comme on pourra le voir sur les photos ef les moulages, la surface extérieure da dépôt forme des vagues sensiblement perpendiculaires a la direction de lécoulement. Les photos 1 et 2 ont ble prises au même endroil de la conduite mélallique, mais la photo 1 est éclairée de l'amont alors que la photo 2 est éclairec de l'aval. La comparaison

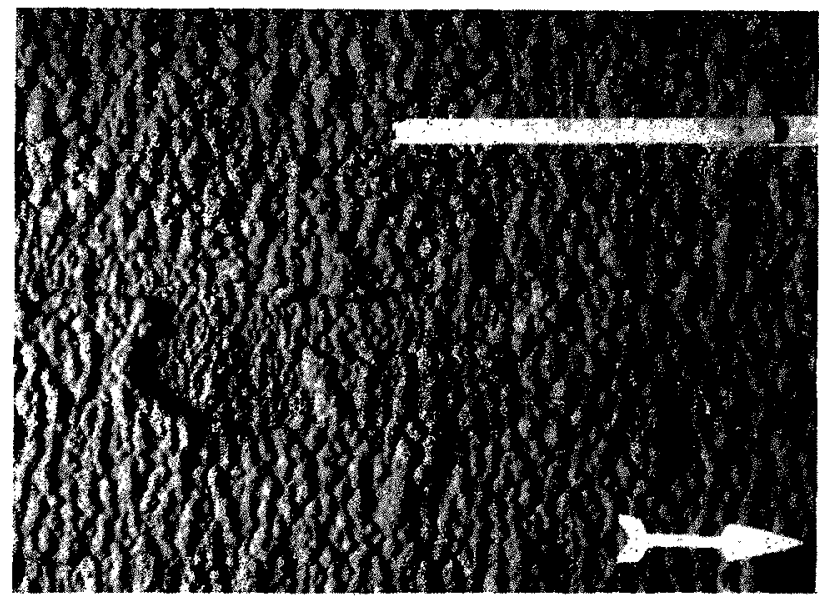

Plom 1 


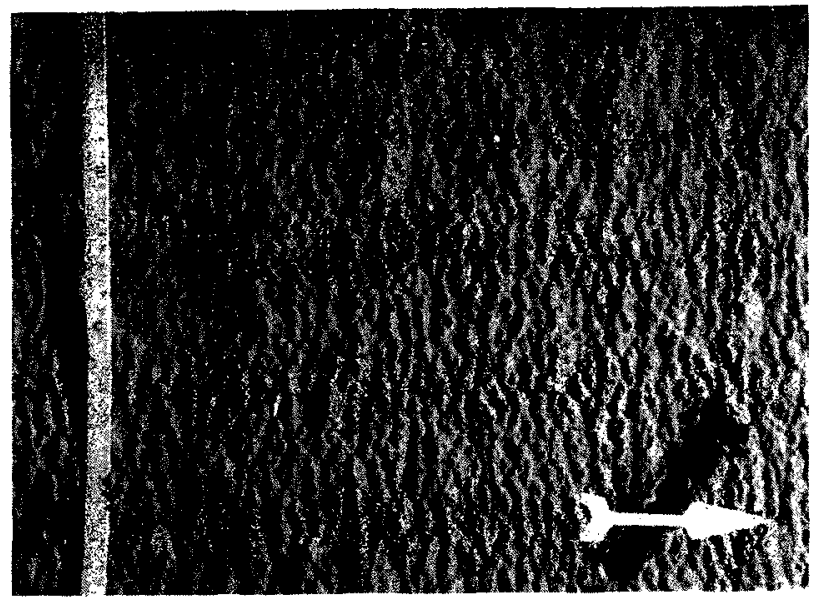

PuOTO2

est en faveur de l'éclairage de l'amont. Aussi loules les autres photos ont-elles ele prises avec un éclairage amont.

Les photos 1 et 2 illustrent une particularité

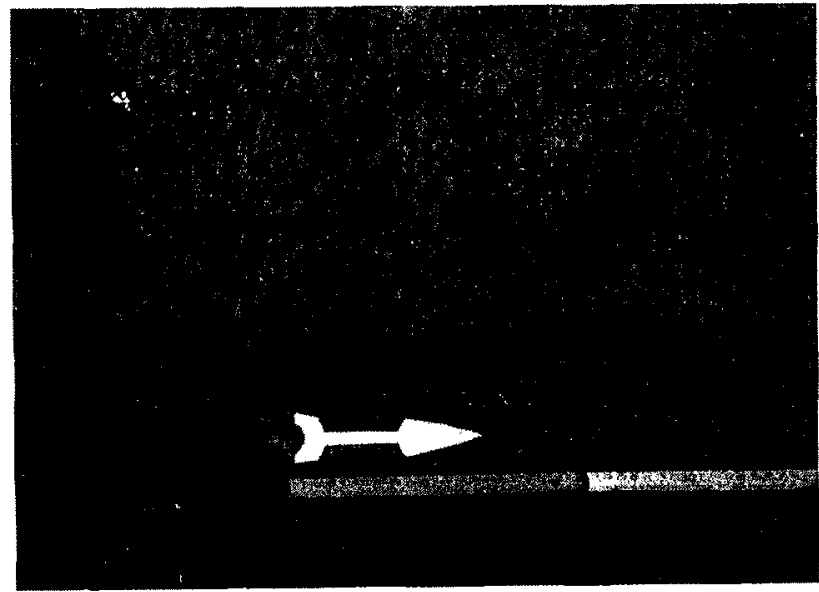

Pното 3

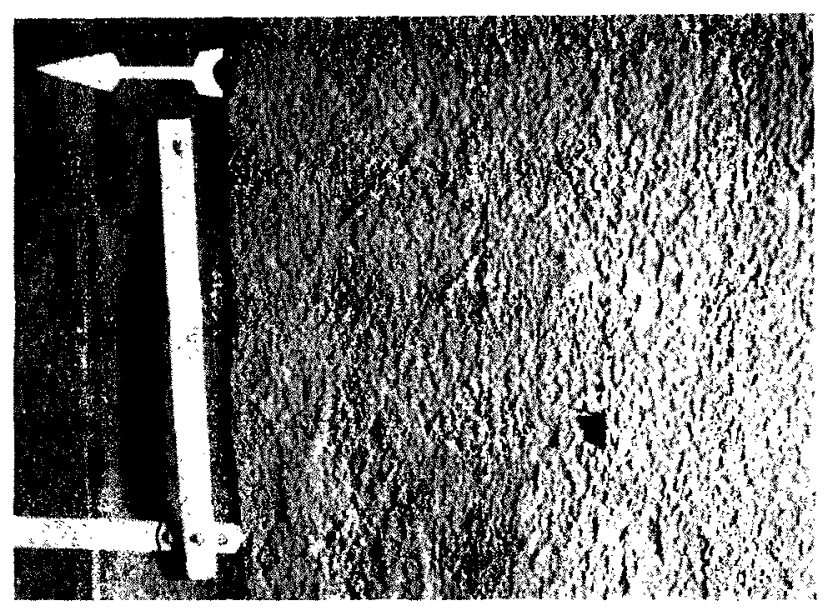

Proto 4 rencontrie assez souvent dans les régions où le dépôt n'a plus été tonché depuis de nombreuses années. L'épaisseur présente des váliations brusques et localisées, comme si des écailles s'en étaient détachées. D'autre part, la longueur d'onde paraît varier avec l'épaisseur. Cette parlicularité est bien visible sur la photo 3 ainsi

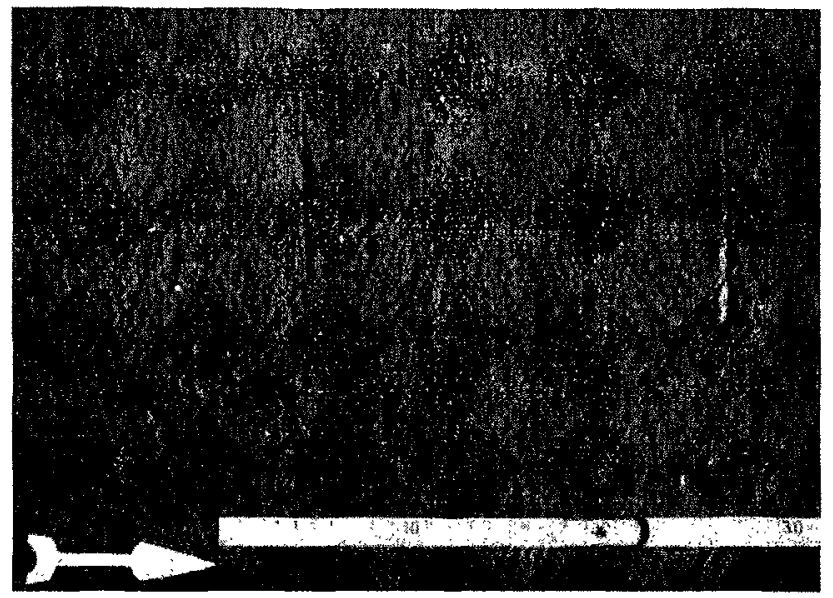

Pното :

que sur le moulage I lail au même endroil. La trace de la boîte à moulage est visible sur la photo.

Dans les conduites en bilon nelloyedes en 1949 , le dépót est très régulier et peu épais, la longueur d'onde est petite (photos 4 et 5 ). Le moulage II pris à l'endroit de la photo 5 comporte

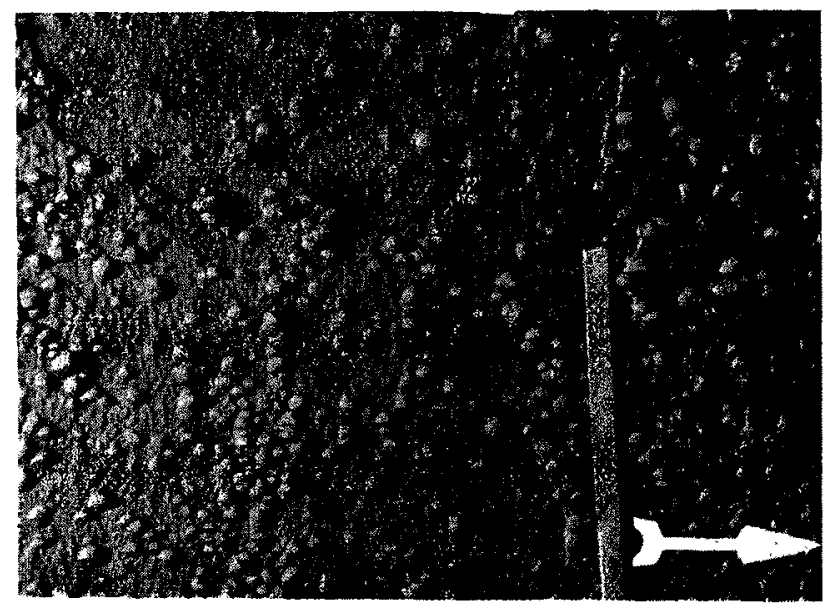

PHOTO ti

une bande moulée jusqu'à la paroi, on aura ainsi une idée assez exacte de l'ćpaisseur du dépôt.

A certains coudes de la conduite, le dépôt présente des traces de charriages et un aspect heaucoup plus lisse, les vagues sont très peu marquées.

Sous le dépôt, Ia conduite métallique était en 
excellent état; le dépôt semble constituer une réelle protection pour la conduite. Les régions les plus dégradées sont les régions supérieures, où le dépôt est moins épais. lhans une zone présentant une profusion de petits nodules de rouille, nous avons constaté que le dépôt citait plus mince (photo 6).

Dans lat partic rocheuse, nelloyece en 1949, le depôt est assez abondant, quoique relativenenl régulier. On ne constate pas d'épaisseurs bealucoup plus fortes dans les fonds des anfractuosités. Il tend à se former une crète de boue sur les saillies. Sur les parties planes, la formation en vagues se retrouve.

Les troncons rocheux ont une section assez tourmentée; les aspérités ont une hauteur de 20 à $40 \mathrm{~cm}$ à partir du contour exlérieur de la section. Celle-ci n'est que très approximativement eirculaire et son diametre moyen est de l'ordre de $2,5 \mathrm{~m}$.

Ces troncons se raecordent a la galerie cimentée, légèrement plus petite, par des trones de cône. Lorsque ces cônes sont divergents relative-

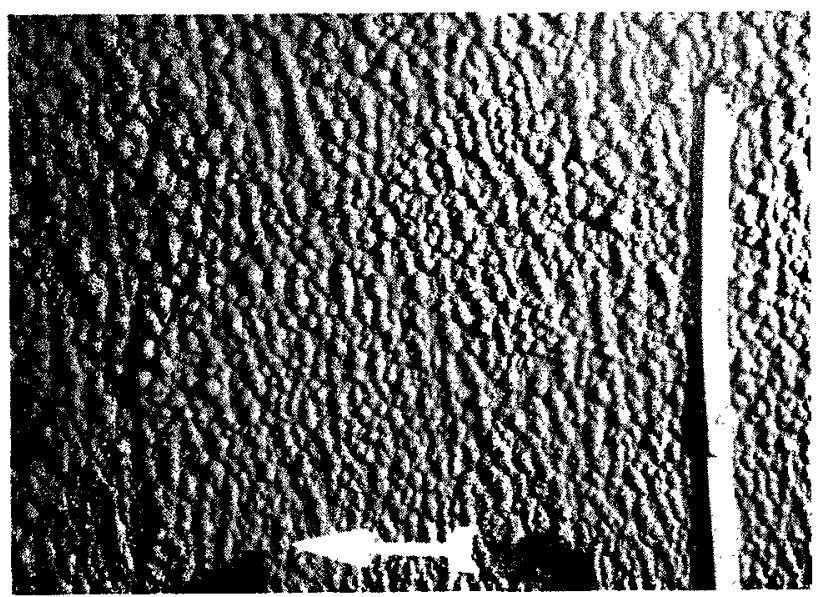

Paroto 7

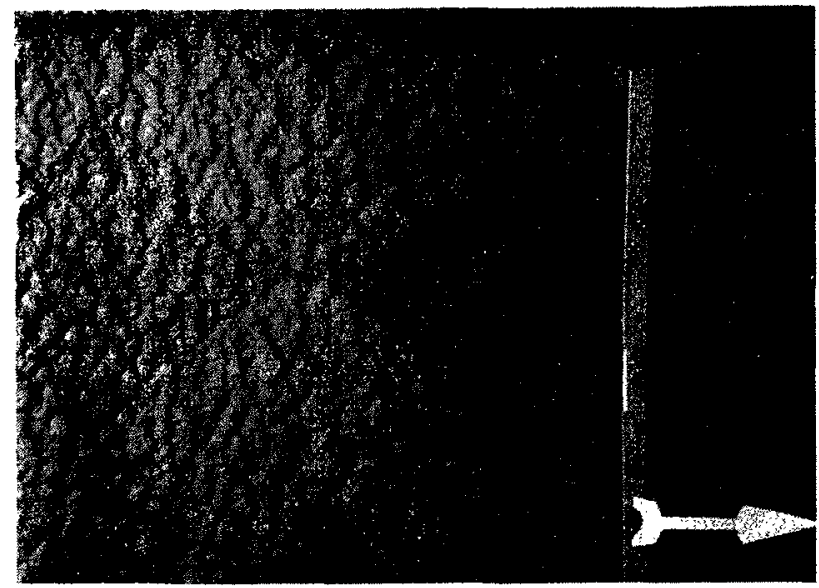

Piroto 8

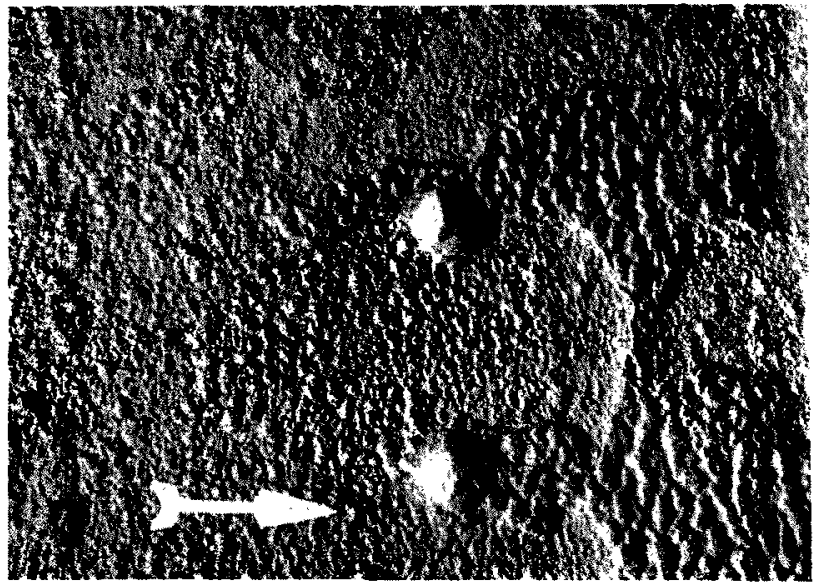

Рното 9

ment au sens de récoulement de l'eau, nous avons constaté que la longueur d'onde du deprit élait de deux a trois fois plus grande que dans la galerie cimentée voisine, el cela sans que l'épaisseur varie de façon appréciable. Nous avons également noté une variation de longueur d'onde semblable entre les parois, extérieures et interieures, de certains coudes de la partie métallique (photos 7 et 8). La variation etail cependant moins importante, de 20 à $25 \%$. Ces variations

Tableau I. - Caractionstroues dis vagues DU DÉPôT

\begin{tabular}{|c|c|c|c|c|c|}
\hline Silnation (") & Paroi & Age & $e$ & $h$ & I. \\
\hline$\Lambda$ & Métallique & 19 ans & 3,5 & 1,25 & 8 \\
\hline$\Lambda$ & Metallicule & 19 : & 0,5 & 2 & 11 \\
\hline$A$ & Matallicfue & 19 ans & $8, \overline{5}$ & $2, \pi$ & 16 \\
\hline Photo 1 & Nétalligue & 17 ans & 12 & 1 & 12 \\
\hline Photo 1 & Nétallique & 17 ans & 7 & 3 & 10 \\
\hline Moulage I & Metallique & $17: 111 \mathrm{~s}$ & !) & 3 & 13 \\
\hline Monlage I & Netallicpure & 17 ams & 2,5 & 1 & i) \\
\hline Moulage III & Netallique & 16 it 18 ans? & 2 & 0,5 & (i \\
\hline Moulage IV & Netallique & I I i : at 18 ans & $9, \bar{i}$ & 3 & 8,5 \\
\hline Monlage $\mathrm{V}$ & Netallique & $\begin{array}{lllll}16 & \text { i } & 18 & \text { ans }\end{array}$ & 8 & 2 & 18 \\
\hline Photo 4 & En ciment & 4 ans & $1, \overline{5}$ & 0,5 & 4,5 \\
\hline Noulage II & En ciment & $f$ ans & 1,5 & 0,4 & 5 \\
\hline
\end{tabular}

(*) Voir fig. 1. 
proviendraient peut-être d'une difference de vitesse à la paroi entre ces regions.

L'observation des différents moulages que nous avons effectues, ainsi que celle des photos, nous montre une forte ressemblance entre l'aspect de la surface du dépòt et celui que l'on peut parfois voir sur les plages de sable. Il rappelle également l'aspect des dunes du désert, analogie qui avait été signale à celte mème lribune par M. Escanot (4).

Ia photo 9 constilue une assez belle synlhese de nos diferentes observations : plage écaillée do faible épaisseur avec nodules de rouille, variation de la longueur d'onde avee l'épaisseur, absence de nodules sous la couche ćpaisse.

Nous arons réuni dans le tableau I lous les iléments relatils aux caractéristiques des vagues formées par le dépôt.

Remarquons tout de suile que ees caractéristiques onl été, soit mesurées au moyen d'un mé tre pliant ordinaire, soit estimée d'après les pholos. On doit donc sattendre à des erreurs qui peurent alteindre facilement $\pm 25 \%$.

Nous avons traduit graphiquement res observations.

D’apres la figure 3 , il semble bien exister une relation entre $h$ ef $e$, relation qui pourait mimc ctre linéaire. En ce qui concerne e el $\mathrm{L}$ ainsi que $h$ et $\mathrm{L}$, il n'est pas possible de conclure dans un sens ou dans l'autre, dautres observations devaient être faites. Quant au diagramme $h / \mathrm{J}_{2}=f\left(e / \mathrm{I}_{\mathrm{s}}\right)$, il corrobore le diagramme $h=f(e)$ tout en presentant l'avantage d'etre sans dimension.

Les données relatives à la conduile de l'Ecker en notre possession ne sont malheureusement pas assez completes pour que nous ayons pu les faire figurer sur nos diagrammes.

Les analyses chimiques effeclues par différents laboratoires du Centre Belge d'Etude el de bocumentation des Eatux ne sont pas uniformes
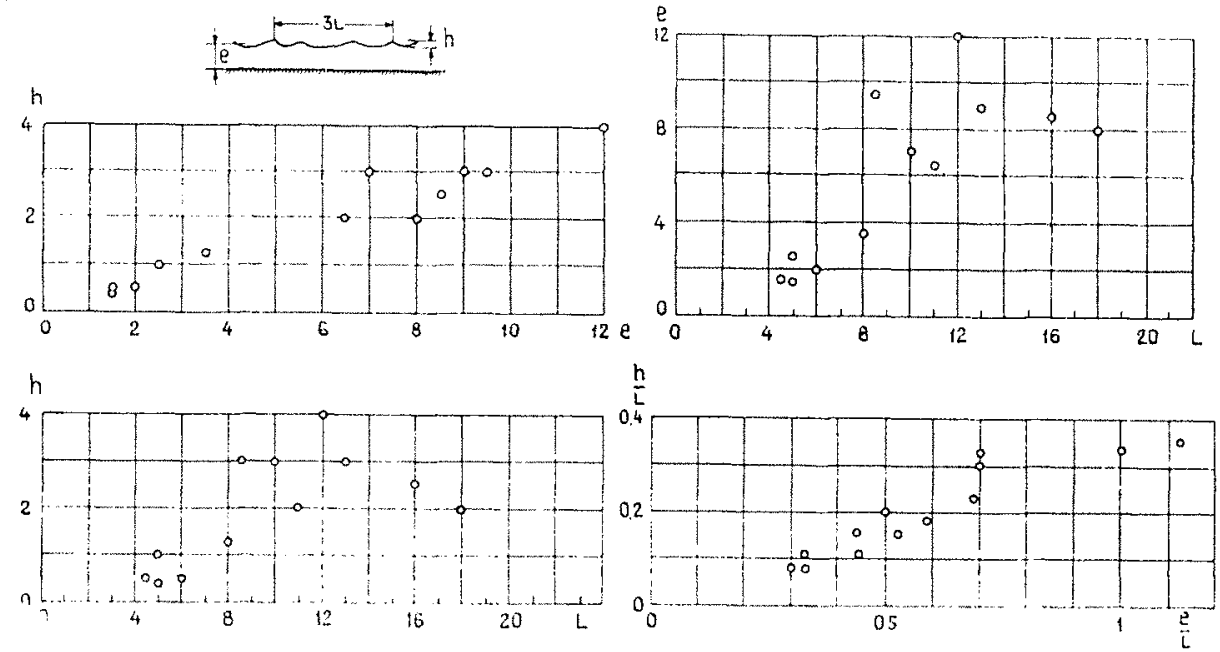

Fig. 3

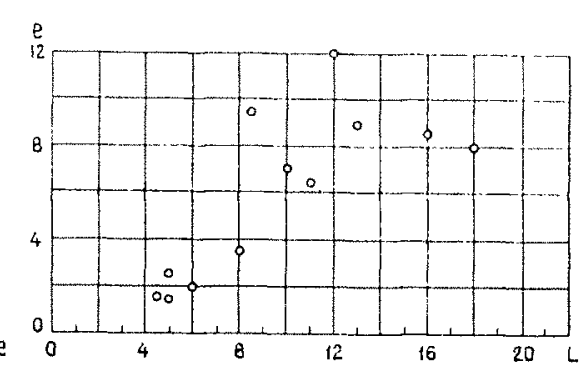

dans leur presentation. La perte au feu et l'humidité de l'échantillon frais n’ont élé dosées que sur un prélevement. Plusicurs échantillons étaient insuffisants pour pouvoir ètre analysés.

Nous avons groupe dans le tableau II les résultats de ces analyses.

La parlicularité de ces chifres est l'énorme

TMbliAU II. -.- ANALYSE CHIMQUe

\begin{tabular}{|c|c|c|c|c|c|}
\hline Echantillon $n "$ : & 28 & 30 & 37 & 38 & nodules \\
\hline Age (on annees). & 19 & 16 a 18 & 4 & 17 & 19 \\
\hline Paroi ...... & Métal. & Métal. & Ciment & Métal. & Métal. \\
\hline Situation $(\cdot) \ldots$ & $A$ & Photo 9) & Pholo th & $\begin{array}{l}\text { en face } \\
\text { Photo } 1\end{array}$ & A \\
\hline $\ln _{3} O_{4} \ldots$ & 56,98 & 55,57 & 56,98 & 56,79 & $\cdots$ \\
\hline$F\left(c_{2}, O_{3} \ldots\right.$ & $3,1 \cdot 1$ & 3.43 & 3,30 & 3,21 & 80,32 \\
\hline Si O... & $\cdots$ & $(0,0)$ & $\cdots$ & & $\ldots$ \\
\hline Cia $0 \ldots$ & Traces & - & Traces & Traces & Traces \\
\hline $\begin{array}{l}\text { Perte au fou.... } \\
\text { Humidite sur }\end{array}$ & - & 29,00 & - & - & - \\
\hline echantillon frais & - & $87 \%$ & $\cdots$ & - & 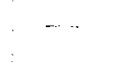 \\
\hline $\mathrm{pH} \ldots \ldots \ldots$ & $\cdots$ & 6 & $\ldots$ & $\ldots$ & $\ldots$ \\
\hline \multicolumn{6}{|c|}{$\begin{array}{l}\text { Analyse sur matière seche a } 110^{\circ} \mathrm{C} \text {, en } \% \text { du poids. } \\
\text { Relicfuat indéterminc. } \\
\text { Analyses effectuees par le Centre Belge d'Etudes et }\end{array}$} \\
\hline
\end{tabular}

(*) Voir fig. 1

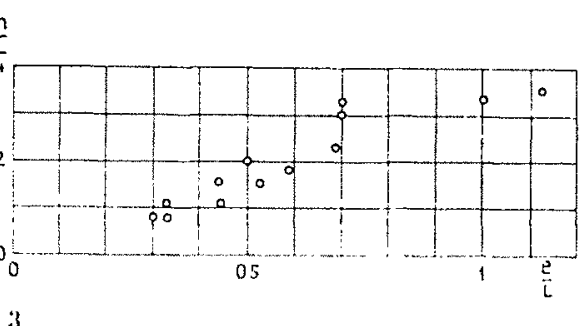


quantité de manganèse renfermé dans le dépơt, $56 \%$, contre $3 \%$ de fer; ct cela, quel que soit le lieu de prélèvement. Par contre, l'analyse d'un nodule de rouille détachí de la conduite métallique, révèle une prépondérance encore plus nette du fer; le manganèse est quasiment absent.

Signalons que M. Vax Bexedex, Directeur du laboratoire des Etablissements des Bains de Spa, s'est déjá beaucoup occupé des dépòts manganifères que l'on rencontre dans les canalisations de l'est de la Belgique, alimentées en eaux de lagnes (5). Lorigine exogene du manganese ne peul en aucun cas étre mise en doute; loulefois, son point de départ, sa mise en suspension, son transport et son depoit sont autant de questions à résoudre.

En ce qui concerne l'étude biologique, notre incompétence nous a conduits à recourir aux lumières des services du professeur BormLLenve, de l'Université de Liège, et à ceux de M. VaN Beneden. De leurs avis, il résulte que le fait le plus marquant à signaler est l'absence quasi totale de cellules organisies; il n'y a pratiquement pas de cellulose dans le dépôt. Cependant, des spores de champignons inférieurs ont été observés en grandes quantités; malheureusement, nous ne savons rien d'eux, ni de leur action.

\section{La stratification}

Lors du moulage du dépòt, une grande partie de celui-ci avait adhéré au plâtre. Une observation attentive nous révila que ce dépót n'est pas constitué d'une maniere homogine. Il se prósente sous forme de couches successives; la strittification est très nette, comme on peut le voir sur la photo 10.

Le décompte des couches, comparé à l'âge du dépôt, nous a donné les résultats suivants (tableau III). Il paraît donc y avoir formation d'une couche annuelle.

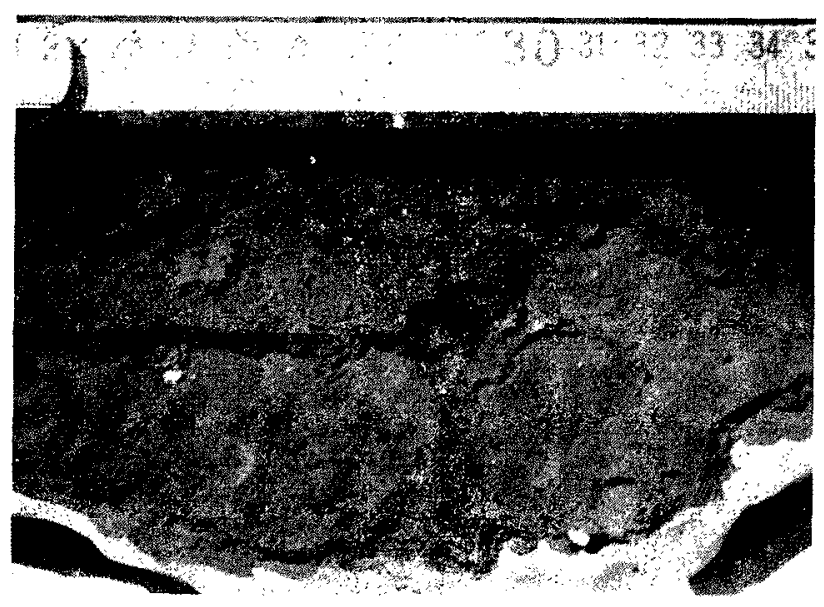

Pnoto 111
Tableitu III. - Stratification

\begin{tabular}{|c|c|c|c|c|}
\hline Situation (*) & Paroi & Age & $\begin{array}{c}\text { Nombre } \\
\text { de } \\
\text { conches }\end{array}$ & Groupement \\
\hline Moulage I & Métallicpue & $17 \mathrm{ans}$ & $16: 18$ & $3+8+6$ \\
\hline Monlage III & Metallique & 16 i 18 ans & $8: 9$ & $\begin{array}{c}\text { Page avec } \\
\text { nodules. }\end{array}$ \\
\hline Moulage IV & Mél:bllique & 16 a 18 ands & 17 & $3+8+6$ \\
\hline Moulage V & Metallique & lli $\vdots 18$ ans & 17 & $(3 a n)+(7$ \\
\hline Moulage II & En ciment & 1 ans & 1 & 4 \\
\hline
\end{tabular}

Poussant plus avant nos investigations, nous avons observé les couches sous un assez fort grossissement de vingt a vingt-eing fois. les couches nous sont apparues héterogènes jusque dans leur épaisscur. De texlure assez lache du

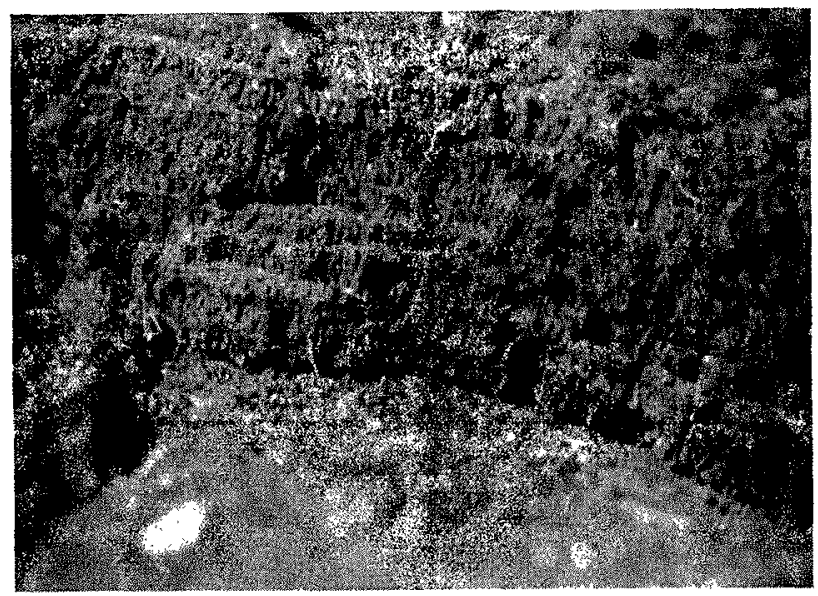

Phoro 11

crite de la paroi, le dípot, qui a un aspect fentré, va en se resserrant du colle de l'eau, justu’i “que l'on rencontre une nouvelle courbe tpholo 11). De plus, la couleur varie d'une couche a laulre, allanl d'un brun ocere asse\% doatud au brun presque noir.

Nous avions pense que celle formation anmuclle pourait etre due a une manifestation faelconque de la vie, soient algues ou mousses, soient micro-organismes. Les specialistes de la question que nous avons interroges ont ecarti d'office les algues of mousses, ba photosynthese neessaire a la vie de ces organismes n'ćtant pals possible dans fobscuriti loblate de la com-

(") Voir fig. 1 

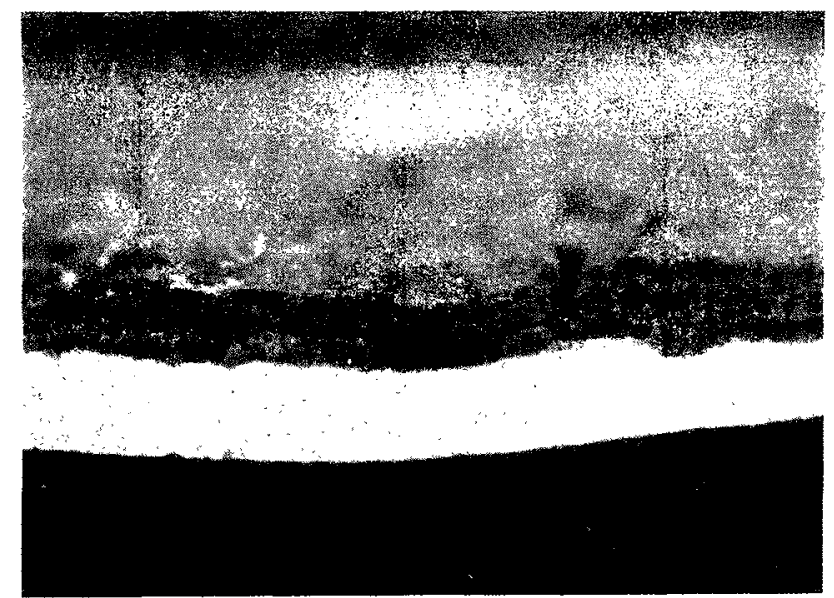

Photo la

duite. Ils nous ont, par contre, signale la présence de champignons inférieurs, mais it n'est pas possible actuellement de relier leur existence $\dot{a}$ la stratification. Celle stratification pose le problème de l'influence du climat sur les phénomènes relatifs aux mouvements du manganèse.

Comme on peut le voir sur la photo 12 , le décompte des couches peut se faire assez aisément. Cependant, un fait, à rapprocher de l'écaillage possible du dépôt, est à signaler. Alors que certaines couches adhèrent bien entre elles, formant ainsi des groupes de couches, d'autres se détachent nettement l'une de l'autre (photo 13). Cette particularité rend le dénombrement total des eouches assez difficile car il faut suivre la

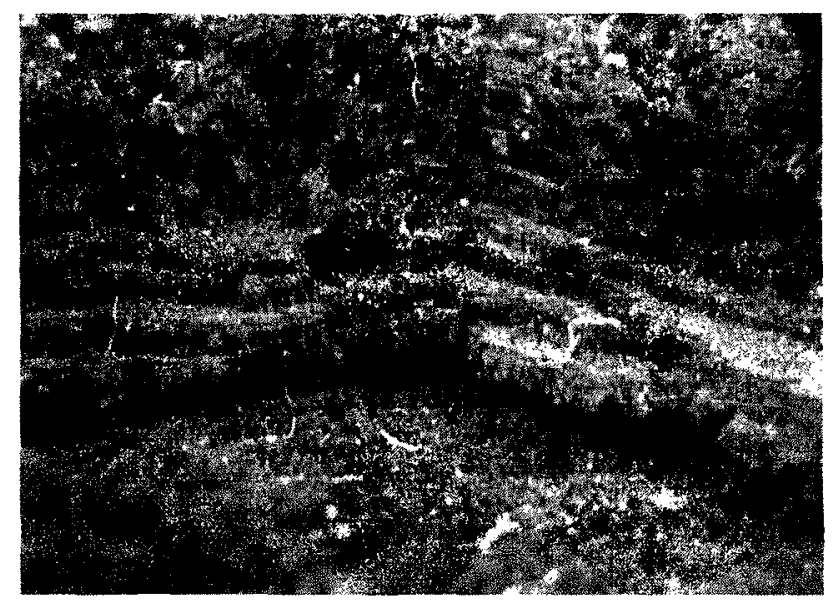

Pното 13 lace d'une couche de séparation parfois assez loin. Aux abords des zones où les couches sont cependant resties accrochées, les coupes ne sont pas toujours bien nettes (photo 14). Le tableau III donne également le groupement des couches a partir de la paroi.

Sans pouvoir etre formels, les biologistes interrogés sur la particularite que pourraient presenter les amnies 1939 et 1947 (qui correspondent aux conches de séparation) iu plateau des fagnes, nous ont signalé que ees périodes ont été particulièrement sèches. Il n'est pas exclu que l'intensité, instantanée ou moyenne, des précipitations ne soit une des données du problème.

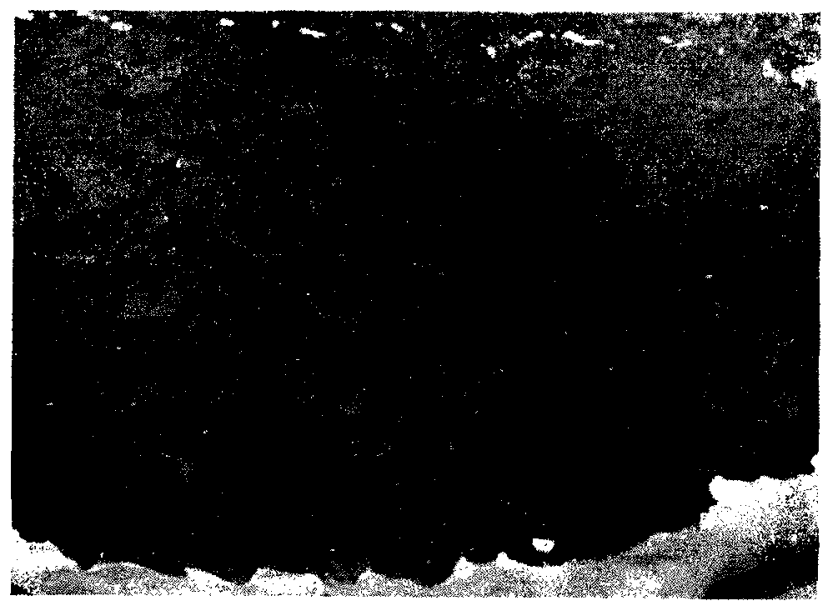

Pното 14

\section{En guise de conclusion}

Ces observations isolées n'ont pas la prétention d'avoir vidé le sujet, notre objectif est uniquement de provoquer la diffusion d'observations analogues qui ont certainement déjà été faites, ('t qui, confrontces, pourraient jeter une lumière plus vive sur cet aspeet particulier du vieillessement des conduites.

De nos observations, trois déments sont à disalger : e/L,

Il y aurait une relation, à definir entre $h / \mathrm{L}$ ol

L pourrait être fonction de la vitesse a la paroi (déjà signalé par M. Escande),

Le dépôt ne se forme pas de façon régulière et continue. 


\section{ANNEXE}

\section{Mode opératoire}

Les photos ont été prises avec un appareil Ikoflex. L'éclairage était fourni par un photollash Gevaert équipé d'ampoules type PF 25. L'obturateur était maintenu ouvert et le temps de pose était le temps de combustion de lat lampe. L'objectif était ouvert à $\mathbf{1} / \mathbf{1 6}$. L'appareil était dans l'axe de la conduite, soit à environ $1 \mathrm{~m}$ de la paroi. Le flash était tenu à environ $2 \mathrm{~m} \dot{\mathrm{a}} 2,5 \mathrm{~m}$ en amont ou en aval de l'appareil photographique, à $30 \mathrm{~cm}$ de l'axe de la conduite du côté où l'on prenait la photo, dirigé vers la zone photographiée.
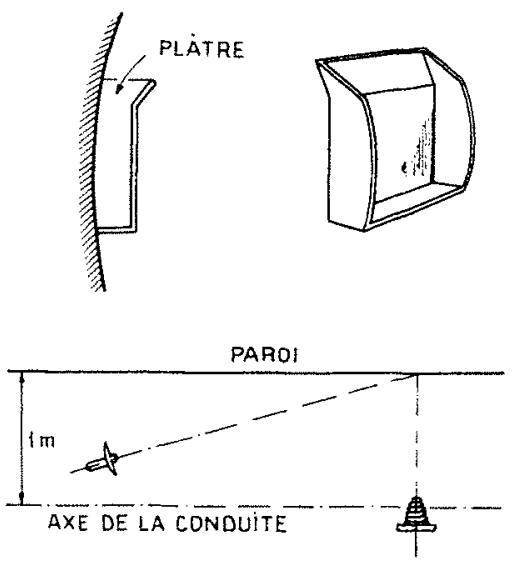

Les moulages ont été réalisés en appliquant une boîte rectangulaire en tôle contre la paroi de la conduite. Les bords verticaux en avaient été tracés plus ou moins suivant le rayon de courbure de la conduite. Le plâtre était coule par le dessus. L'étanchéité étail assurée par le dépòt lui-mème dans lequel la boite était enfoncée au maximum (pratiquement jusqu'à la paroi). Le plâtre utilisé élait du plâtre de dentiste, délayé dans de l'eau légèrement saléc jusqu'à la consistance d'une huile fluide. Il était alors baltu jusqu'au moment où la consistance commençait à varier. Cet instant doit être sassi avec précision, car, à une minute prés, le plâtre n'est plus utilisable. Il était enfin coulé dans la boîte. Nous avons utilisé avec succès, comme récipient pour gâcher le plâtre, une balle en caroutchouc de $30 \mathrm{~cm}$ de diamètre environ, coupée suivant un méridien. Ce récipient est d'un emploi très commode pour autant, toutefois, que les parois en soient suffisamment épaisses (3 à $4 \mathrm{~mm}$ ).

\section{Bibliographie}

(1) Alb. Schlag et R. Simons. - Variations de la perte de charge du canal d'amenée de la centrale de Bévercé. Mémoires et Travaux de la S.H.F., 1951, n 2, p. 149

(2) Schurchrisg. - Experimentelle Untersuchungen zum Rauhigkeitsproblem. Ingenieur-Archiv, vol. VII, n' 1, 1936, p. 1-34.

(3) R. SEIFERTH et W. Knugith. - Ueberraschend hohe Reibungsziffer einer Fernwasserleitung. Z.V.D.I., vol. $92, \mathrm{n}^{\circ} 8,11$ mars 1950 , p. 189.

(4) L. Escande. - Similitude des ondulations de sable des modèles réduits et des dunes du désert. Mémoires et Travaux de la S.H.F., 1950, $\mathrm{n}^{\circ} 1, \mathrm{p} .87-92$.

(5) G. Van Benedes. - Communication sur les dépôts at incrustations des réseaux de l'est de la Belgique. (A paraître dans Bulletin du Centre Belge d'Etude et de Documentation des Eaux. en fin 1954).

\section{I S C U S S I O N}

Président: M. Bannition

M. le Président souligne l'intérêt de telles communications sur les observations de conduites sales et la valeur de l'exposé de M. Thibessand, notamment en ce qui concerne l'évolution des dépóts : la différence d'aspect des dépôts à l'intéricur et à l'extéricur d'un coude est une observation nouvelle; et la différence éventuclle des dépôts dans les parties convergentes et dans les partics divergentes serait également intéressante à rechercher par ceux qui ont l'occasion d'observer des dépots dans des conditions variées.

M. Ferny rend hommage à la minutie et à la précision de ces observations. Il indique que les dépôts gélatineux que son service a observés dans des conduites en exploitation dans le Massif Central depuis deux ou trois aus causent des pertes de charge analogues à celle de II. Thubssarn, mais ces dépôts sont à base de fer (baclérie du fer et non de manganèse : l'épaisseur des dépôts ne dépasse pas quelques millimètres et l'attention du service n'avail pas eté altiréce jusqu'à présent sur la phénomène de ciratification signalé par M. Tumussam, La loi de croissance du dépôt avec la saison va elre dudiée sur modèle réduit en utilisant des buses trempéce dans l'eau du canal de fuite et soumises périodiquenent a des essais de pertes de charge ainsi qu'à divers traitements. Il est probable que la croissance est moins rapide sur une buse neuve que sur une buse déja ensemencée puis nettoyée, le netoiement ne détruisant pas la tot:a lité des micro-organismes.

En réponse à une question de $M$. le Président, M. TH IESSARD indique que la ville de Malmédy est alimentéc en eau par la conduite de Bévercé, à travers une station de traitement, mais qu'il ignore si des constatations particulières ont été faites sur les dépôts de manganése dans le réseau de distribution.

Il signale, toutefois, que de tels dépots ont ite constatés dans différents ourrages hydrauliques reliant le 
réservoir de la Gileppe à la ville de Verviers, installations situées dans la même région. Le dépôt ne paraîl pas influencé par la nature de la paroi sur laquelle il se forme : on a, en effet, trouvé sur des planches, non des nodules, mais des lentilles de manganèse tortement développées. Il semble également que les dépôts soient plus importants en tête qu'en fin de réseau. Cet aspect du problème sera évoqué par M. Van Benedes dans un article qu'il publiera dans le Bulletin du Centre belge d'Etude et de Documentation des Eaux.

I. le Président suggère une comparaison entre ces dépóts et ceux observés par M. Thibessard au point de vue de la viscosité et de l'infuence de la pression.

Sur la demande de M. Gibert, M. Thinessard indique qu'il a constaté la variation de la schistosité des dépôts, que celle-ci semble varier d'années en années et, peutêtre aussi au cours des saisons mêmes, mais sans pouvoir tirer une conclusion sur l'allure et la cause de cette variation.

M. LangloIs souligne l'intérêt de la constatation relalive a la formation annuelle et non continuelle des depôts, et appuie les remarques et les projets d'études analogues de M. Ferry.

M. Escande demande si la périodicité de ces phéno- mènes n'est pas duc à ce que les eaux sont chargées dans certaines saisons et pures dans d'autres.

M. Thibessamo répond qu'une telle hypothèse est raisonnable, mais n'a pu être vérifiée, la campagne d'observations ayant été faite en septembre et octobre 1953, et n'ayant porti que sur la conduite vide, mais que M. VaN RENEDEx at pu, au sujet de la matière déposéc, vérifier assez grossièrement la constance de l'épaisseur du dépôl en comparant le poids d'échantillons de $20 \mathrm{~cm}$ sur $20 \mathrm{~cm}$ prélevés en quelques endroits de la conduite, an poids total de matières enlevées lors du nettoyage.

M. Turc souligne que ces dépôts sont remarquables par leur haute teneur en manganèse, très supérieure à leur teneur en fer; il est possible que l'explication de co fait soit donnée par une étude pédologique de la région. Au Laboratoire des Sols, M. Betremieux a montré expérimentalement l'importauce des processus biologiques dans les phénomènes de mobilisation, migration, et accumulation, du fer et du manganèse dans les sols; il a précisé que lorsque les conditions sont favorables à la formation de dépôts (arrivẻe des eaux chargées en fer et en manganèse dans un milieu plus aéré) le fer précipite plus rapidement que le manganèse.

M. le Président dit à M. Thubessari, en le remerciant. qu'il a intéressé tout le Comité Technique.

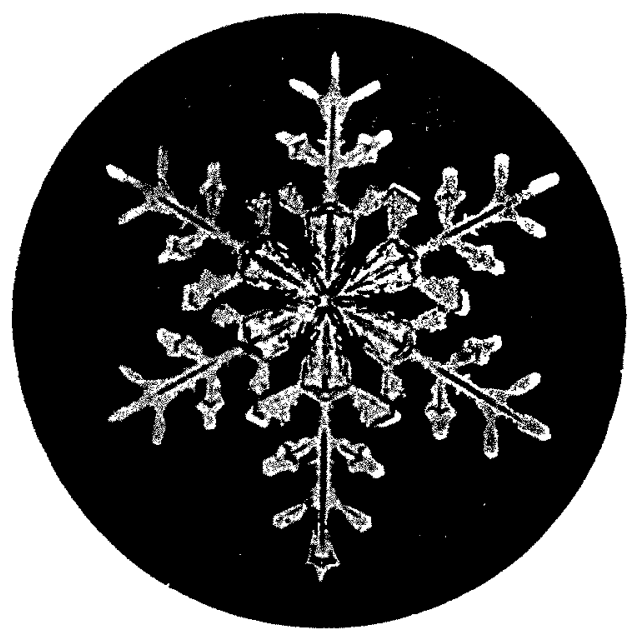

